\title{
The future of $\mathrm{SCl}$ rehabilitation: Understanding the impact of exoskeletons on gait mechanics
}

\author{
Ashraf S. Gorgey ${ }^{1,2}$, Matthew E. Holman $\oplus^{1,3}$
}

${ }^{1}$ Spinal Cord Injury and Disorders, Hunter Holmes McGuire VA Medical Center, Richmond, Virginia, USA, ${ }^{2}$ Physical Medicine and Rehabilitation, Virginia Commonwealth University, Richmond, Virginia, USA, ${ }^{3}$ Physical Therapy, Virginia Commonwealth University, Richmond, Virginia, USA

We currently live an era in which science fiction movies are being translated into real rehabilitation approaches for persons with disabilities. Nowhere is this more evident than with the adoption of robotic exoskeletons in the rehabilitation of persons with spinal cord injury (SCI). This tool evolved over years of multidisciplinary research among teams of biomedical engineers, computer scientists, informatic specialists and gait analysts. ${ }^{1}$ Unlike other available locomotor interventions, exoskeletons offer independence in standing and walking with very low metabolic cost, while also facilitating improvement in the users' quality of life. ${ }^{1-3}$ However, little is known about the neuromechanical adaptions and temporal-spatial gait characteristics of robotic exoskeleton ambulation in persons with SCI.

This editorial references two articles published in the Journal of Spinal Cord Medicine that highlight the effects of exoskeleton use on the temporal-spatial, kinematic, and neuromechanical components of walking. ${ }^{4-5}$ Improving our understanding of how exoskeleton use and training duration impacts walking mechanics is fundamental to the development of improved rehabilitation strategies for individuals with SCI; additionally, it can expand our grasp of the underlying mechanisms involved in improving locomotor function in persons with SCI. This knowledge may also influence the development of future powered robotic exoskeletons that enhance neuromuscular function in persons with SCI.

The first article by Hayes et al. ${ }^{4}$ is a systematic review comparing the effects of robotic assisted gait training (RAGT) over a treadmill and RAGT training overground on the temporal-spatial components of

Correspondence to: Ashraf S. Gorgey, MPT, PhD, FACSM, Chief of Spinal Cord Injury Research, Hunter Holmes McGuire VA Medical Center, Spinal Cord Injury \& Disorders Service, 1201 Broad Rock Blvd, Richmond, VA 23249, USA; Ph: 804-675-5000 ext 3386; Fax: 804-675-5223. Email: ashraf.gorgey@va.gov walking for individuals with SCI. The authors identified 12 studies that met their inclusion criteria. Of these, $30 \%$ were considered randomized controlled clinical trials, and information for both sexes was provided. The majority of participants had incomplete SCI classified as International Standards for Neurological Classification of Spinal Cord Injury (ISNCSCI) C-D. Only two trials were included for individuals with complete injuries classified as ISNCSCI A.

Hayes $\mathrm{et} \mathrm{al}^{4}{ }^{4}$ surmised that for individuals with SCI, RAGT can result in positive neuronal adaptations, improved temporal-spatial gait mechanics, and decreases in energetic costs associated with walking (compared to traditional rehabilitation methods). The group also noted that some strength increases are possible among persons with incomplete SCI when resistance is integrated into RAGT. They also suggested that RAGT may result in individuals spending more time in upright postures which may ameliorate several of the negative health consequences of sitting in wheelchairs, as well as providing the opportunity for some neural recovery for persons with incomplete SCI.

When considering the mechanics of normal gait, the group also identified some benefits specific to the over-ground modalities, as these require additional balance and support from the rest of the body. A certain amount of gait variability occurs normally within able-bodied (AB) populations and treadmill devices normalize stepping patterns; over-ground devices provide for more gait fluctuations, more closely mirroring $\mathrm{AB}$ populations. The authors concluded that there were no significant differences between RAGT treadmill walking speed and distance compared to conventional over-ground ambulation training, as walking speeds identified ranged from 0.31 to $0.4 \mathrm{~m} / \mathrm{sec}$. Variations among and within the study populations paired with the limited number of subjects 
across the articles make definitive results of their review difficult at best. However, the group carefully considered all of the literature to identify overarching trends, while also providing guidance for future studies. Their analysis indicates that RAGT as an upright ambulatory aid utilizing either modality is helpful for both incomplete and complete SCI rehabilitation, especially when integrated into a more comprehensive and balanced rehabilitation regimen that considers the individual's needs and capabilities. ${ }^{4}$

In their exploratory case series, Ramanujam et $a l^{5}$ observed four $\mathrm{AB}$ controls and four persons with $\mathrm{SCI}$ ( 2 complete, 2 incomplete) during different gaiting conditions within a single session. The $\mathrm{AB}$ individuals walked unassisted over-ground at three speeds and while utilizing an exoskeleton under both passive and active control; the participants with SCI walked under active exoskeleton control only. The group provided a thorough evaluation of each subject's temporal-spatial gait mechanics, lower extremity sagittal plane kinematics, and neuromuscular activity using a passive, retroreflective 3D-marker system in conjunction with surface electromyography (EMG).

The data clearly indicated that the $\mathrm{AB}$ individuals walked differently than the individuals with SCI, as they exhibited different temporal-spatial and muscle activation patterns when walking both with and without an exoskeleton. ${ }^{5}$ However, sagittal plane range of motion for both the knee and hip joints were not different between the SCI and $\mathrm{AB}$ groups during their normal walking conditions. Among the walking conditions completed by the $\mathrm{AB}$ control group, the passive exoskeleton ambulation condition proved to be most comparable to the SCI group. This is most evident in the percentage of stance time between the SCI subjects $(86.8 \%)$ and the passive exoskeleton $(79.6 \%)$. While stance time did not change, walking velocity was greater for the $\mathrm{AB}$ controls even when passively moved by the exoskeleton $(0.25 \mathrm{~m} / \mathrm{sec})$ when compared to the SCI subjects $(0.13 \mathrm{~m} / \mathrm{sec})$. Notably however, the SCI subject with the most exoskeleton training walked with a velocity much higher than the other individuals with SCI $(0.27 \mathrm{~m} / \mathrm{sec})$, which was much closer to the velocity for the $\mathrm{AB}$ control group. ${ }^{5}$

$A B$ individuals adopted different muscle activation patterns in their lower extremities while wearing the exoskeleton, which was primarily evidenced through differences observed in the amplitude and duration of musculature activation of the hip and knee. The SCI subjects on the other hand displayed both inconsistent muscle durations, as well as smaller muscle amplitudes, compared to $\mathrm{AB}$ controls. The investigators suggested that these observed muscle activation patterns in both the $\mathrm{AB}$ group while utilizing the exoskeleton as well as the SCI group may be a result of afferent responses to activation of the stretch reflexes and joint loading. Interestingly, the same individual with SCI who received the most exoskeleton training appeared to display more normal duration patterns.

Sweeping conclusions about the SCI gaiting patterns, either mechanically or neuromechanically, were ultimately not possible due to the limited number of SCI subjects, inconsistencies within the amount of exoskeleton training received by each individual with $\mathrm{SCI}$, as well as the heterogeneity of the SCI demographics (injury and otherwise). This said, training did appear to improve one SCI subject's ability to ambulate, as his gait symmetry, velocity, and neuromuscular activation improved. The authors attributed these neuromuscular adaptions to the repetitive loading and reciprocal movement of the lower extremities within the exoskeleton.

Both reports highlight that robotic exoskeleton research is still in its infancy and significant strides are needed to overcome several of the highlighted limitations. However, despite the limited availability of information detailing exoskeleton use in SCI rehabilitation, it does appear promising as adjunct tool for the rehabilitation team. More research investigating the gait and neuromechanical changes observed with exoskeleton use among individuals with SCI is desperately needed, as are studies examining the effect of training dosage and community use. While we may still be short of a science fiction future where exoskeletons enable their users to ambulate just as well (if not better) than their $\mathrm{AB}$ counterparts, with continued advances in research and technology, reality seems to be catching up quickly.

\section{Disclaimer statements}

Conflicts of interest The authors have no conflict of interest to declare.

\section{ORCID}

Ashraf S. Gorgey (10) http://orcid.org/0000-0002-91576034

Matthew E. Holman (1) http://orcid.org/0000-00019734-2418

\section{References}

1 Gorgey A, Sumrell R, Goetz L. Exoskeletal assisted rehabilitation after spinal cord injury. In: Atlas of Orthoses and Assistive Devices, 5e. Canada: ELSEVIER; 2018. P. 440-47.

2 Miller LE, Zimmermann AK, Herbert WG. Clinical effectiveness and safety of powered exoskeleton-assisted walking in patients with spinal cord injury: systematic review with meta-analysis. Med Devices (Auckl) 2016;9:455-66. 
3 Gorgey A, Wade R, Sumrell R, Villadelgado L, Khalil R, Lavis T. Exoskeleton training may improve level of physical activity after spinal cord injury. A case series. Top Spinal Cord Inj Rehabil 2017;23(3):245-55.

4 Hayes SC, James Wilcox CR, Forbes White HS, Vanicek N. The effects of robot assisted gait training on temporal-spatial characteristics of people with spinal cord injuries: A systematic review. J
Spinal Cord Med 2018; Epublished 5 Feb 2018 DOI:10.1080/ 10790268.2018.1426236

5 Ramanujam A, Cirnigliaro CM, Garbarini E, Asselin P, Pilkar R, Forrest GF. Neuromechanical adaptations during a robotic powered exoskeleton assisted walking session. J Spinal Cord Med 2017; epublished 20 April 2017 DOI:10.1080/10790268.2017. 1314900 